\title{
Preparation of Multi nitrate molten salt and its properties tests
}

\author{
Wei Zhai *, Bo Yang, Maodong Li, Shiping Li, Mingliang Xin, Jinmei Lin, Lian Wang \\ Guangzhou Special Pressure Equipment Inspection and Research Institute,510100 Guangzhou, China
}

\begin{abstract}
In this paper, by adding different additives in Solar Two molten salt in order to form multiple composite molten salts. And testing the thermal performance of mixture molten salt can be comparatively analysis of the modification effects of different additives. The melting point and latent heat of mixture molten salts were characterized by DSC. Through experimental and analysis results showed that additive-A can optimize the melting point and heat of fusion of molten salts, Additive-B and Additive-C have good effect only in melting point or melting heat unilaterally. By researching and improving performance of molten nitrate in order to promote the molten nitrate which play a more important role in thermal power generation and environmental
\end{abstract}

\section{Introduction}

In recent years, as oil prices rise and countries increasing demand for energy in the world. Solar energy, a kind of renewable energy, could resolve the energy shortage problem. Solar thermal power, solar hydrogen and other new energy development projects become a hot research. In the field of solar thermal power, due to the instability of solar energy, excellent high-temperature heat regenerative refrigerant become the key to ensuring a solar thermal power system working properly.

In the field of high-temperature thermal solar energy utilization, the common heat-accumulating material mainly water/steam, oil, liquid metal, air and high temperature molten salt, etc[1]. However, the air as the heat transfer and thermal storage medium which has small of specific heat capacity and caloric density. Liquid metal has a high cost, a highly toxic; it is also limited scope. In addition, oil exists easily ignited, easy to produce toxic vapors, expensive, short life and other Shortcomings. Compared with Several front heat transfer and thermal storage medium, the molten salt have a low melting point, melting heat large, high specific heat capacity, low viscosity, low corrosion and good thermal stability. Molten salts have unusual solvent properties which have led to their use in electrochemistry and metallurgical electrolysis. In the industry, hightemperature molten salt as the heat transfer thermal storage medium more used widely.

At present, molten salt has been widely applied in the field of energy[2-8].The heat transfer medium in solar thermal power stations is usually used by potassium nitrate, sodium nitrate, calcium nitrate and their mixtures and they have been used successfully as thermal energy collection and storage fluid in a large-scale. The molten salt have used been as a heat thermal storage medium in a large-scale. The Nitrate molten salt system has melting heat large, high specific heat capacity, low viscosity, low corrosion and good thermal stability and easy to get. In addition, the mixture nitrate molten has good thermal stability at a high temperatures, the corrosion of containers and piping material in the molten salt is small, particularly suitable for high-temperature thermal storage medium heat.

In foreign, Solar Two binary molten salt, Hitec ternary molten salt are mainly used as heat transfer and heat storage medium in the solar thermal power plant. But Solar Two molten salt has a high melting point disadvantage and Hitec molten salt is easy to cracking the problem of decomposition. Alexander J Jr [9] and Kirst W E [10] studies show that Hitec molten salt has good chemical stability below $454.4^{\circ} \mathrm{C}$. Silverman M D[11] study found that ternary molten upper limit temperature is $450^{\circ} \mathrm{C}$. Yu Jianguo and other invented a binary molten salt which is added to $\mathrm{LiNO}_{3}$ in ternary molten salt basis. So the molten salt temperature range widened to $250^{\circ} \mathrm{C}$ $\sim 550^{\circ} \mathrm{C}$. Due to the addition $\mathrm{LiNO}_{3}$ increases the cost of molten salt, container and pipeline systems, it is used industrially subject to certain restrictions. The researchers hope to get a molten salt having a low melting point, melting heat large, wide temperature range, low corrosion, inexpensive and easy to get.

In this paper, by adding different additives in Solar Two molten salt in order to form multiple composite molten salts. By testing the thermal performance of mixture molten salt can be comparatively analysis of the modification effects of different additives. Compare to modification effect of different additives, it is preferable that the best modification effect of additive and the amount of additive. The heat capacity, density, viscosity, thermal conductivity of the good thermal performance of mixture molten salts were characterized.

\section{Experimental}

The mixture molten salts were prepared by mixing Solar Two, additive-A, additive- $\mathrm{B}$, or additive-C. The mixture molten salts preparation program is shown in Table 1 . The additive-A, additive- $\mathrm{B}$ and additive-C were obtained from samples with Longxi chemical plant without further purification and they were dried in an air oven at $120^{\circ} \mathrm{C}$ for $24 \mathrm{~h}$. Molten salts were put into a powder mill and mixed uniformly in a macroscopic scale by mechanical rolling. Subsequently, these molten salts were placed in an muffle furnace, statically heated to melt completely at $400{ }^{\circ} \mathrm{C}$, kept for $60-120 \mathrm{~min}$ and then cooled to room temperature naturally. Finally, the cooled solids were ground to a powder and then the powder was mixed by mechanical rolling in a powder mill again. Meanwhile, the power was sealed and kept in a dryer. 
Table 1. Additives added programs

\begin{tabular}{|c|c|c|c|}
\hline & additive-A & additive-B & additive-C \\
\hline \multirow{2}{*}{$\begin{array}{c}\text { amount } \\
\text { of } \\
\text { additive }\end{array}$} & $2 \%$ & $5 \%$ & $2 \%$ \\
\cline { 2 - 4 } & $10 \%$ & $10 \%$ & $5 \%$ \\
\cline { 2 - 4 } & & & $10 \%$ \\
\hline
\end{tabular}

Besides, Thermogravimetry-Differential Scanning Calorimetry instrument STA-409PC produced by German NETZSCH Company, was used to measure mixture molten salt's melting point. The molten specific heat was measured by DSC. The measurements conditions for the sample, the reference (a sample with a known specific heat capacity, for example, sapphire) and the empty pan were the same. The specific heat capacity of the sample was calculated by Eq.(1) from the DSC data obtained.

$$
C p_{S}=\frac{H m_{r}}{h m_{s}} C p_{r}
$$

where Cps, specific heat capacity of sample ( $\mathrm{kJ} / \mathrm{kg} \mathrm{K}$ ), $\mathrm{Cpr}$,specific heat capacity of reference $(\mathrm{kJ} / \mathrm{kg} \mathrm{K}), \mathrm{ms}$, mass of sample $(\mathrm{kg}), \mathrm{mr}$, mass of reference $(\mathrm{kg}), \mathrm{H}$,DSC temperature difference of sample and empty pan, h, DSC temperature difference of reference and empty pan.

Density measurements were carried out using a standard densitometer which has fixed volume. Initial weight of the densitometer was measured. Salt mixture held in a beaker and the empty densitometer were both placed on an insulated hot plate. The temperature was set to a fixed value above the melting point of the salt and was measured by a K-type thermocouple. The temperatures of empty densitometer and that of salt were measured separately by K-type thermocouples. After the set temperature was reached and showed identical and stable reading, the molten salt was poured in to the densitometer up to the set mark on the bottle that refers to a fixed volume. The weight of the salt mixture is determined by using an electronic weighing balance. The density of the salt mixture at a given temperature is calculated from the weight of the salt mixture and the fixed volume of the densitometer.

The thermal conductivity of molten salt was measured by laser flash method. The viscosity was measure by in Rheotronic II Viscometer (America TA companies).

\section{Results and discussion}

\subsection{Melting point and latent heat of mixture molten salts}

The mixture molten salts were prepared by mixing Solar Two, additive- $\mathrm{A}$, or additive- $\mathrm{B}$, or additive-C. The melting point and latent heat of molten salts were important characteristics for thermal storage materials.
The melting point and latent heat of molten salts were researched by DSC. In Figure 1 is DSC curve of Solar Two molten salt. DSC results show that the melting temperature is $220.8^{\circ} \mathrm{C}$ and phase change latent heat is $95.6 \mathrm{~J} / \mathrm{g}$.

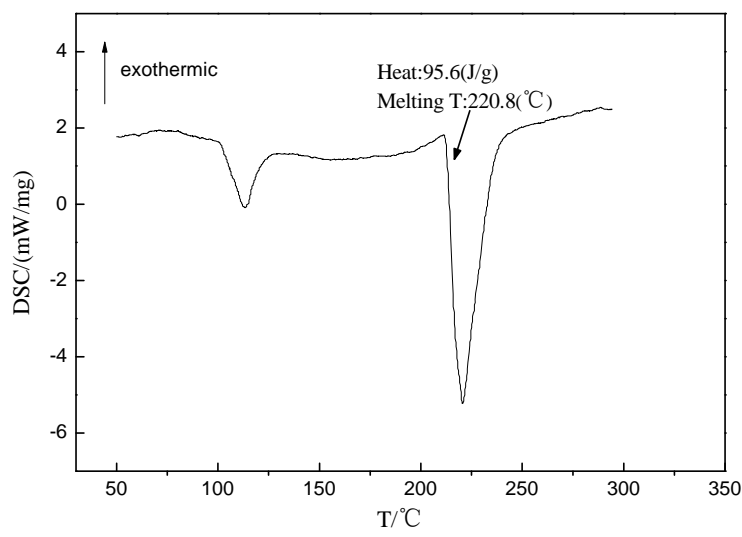

Figure 1. DSC curve of Solar Two molten salt

In Figure 2-9 are DSC curve of mixture molten salts. Figure 2-4 shows the DSC curve of Solar Two +2\%additive-A,5\%additive-A,10\%additive-A. The figure shows that when adding $2 \%, 5 \%, 10 \%$ additive- $A$, the melting point of mixture molten salts were lower, the melting heat were increased. With the increase of the amount of additive-A, the melting point of molten is the greater decline, its heat of fusion is the greater increase. When adding $10 \%$ additive- $\mathrm{A}$, the melting point of Solar Two+2\%additive-A is $205.6{ }^{\circ} \mathrm{C}$, heat of fusion is $113.23 \mathrm{~J} / \mathrm{g}$, it has the best thermal performance.

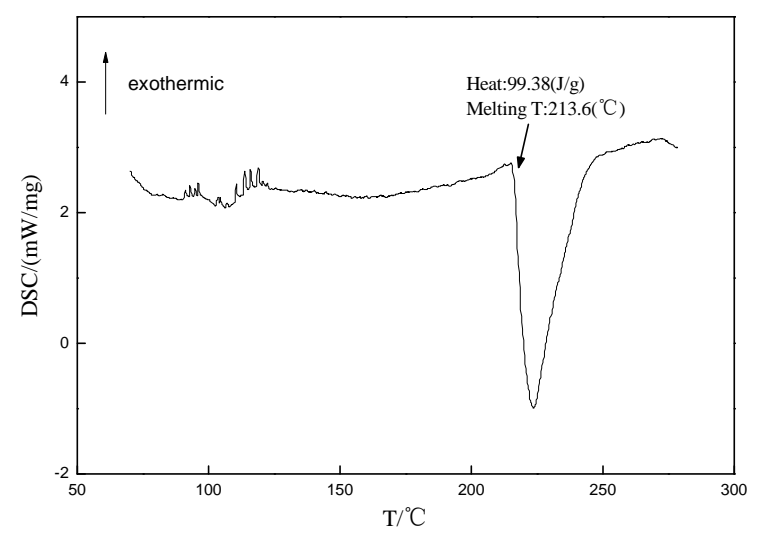

Figure 2. DSC curve of Solar Two+2\%additive-A 


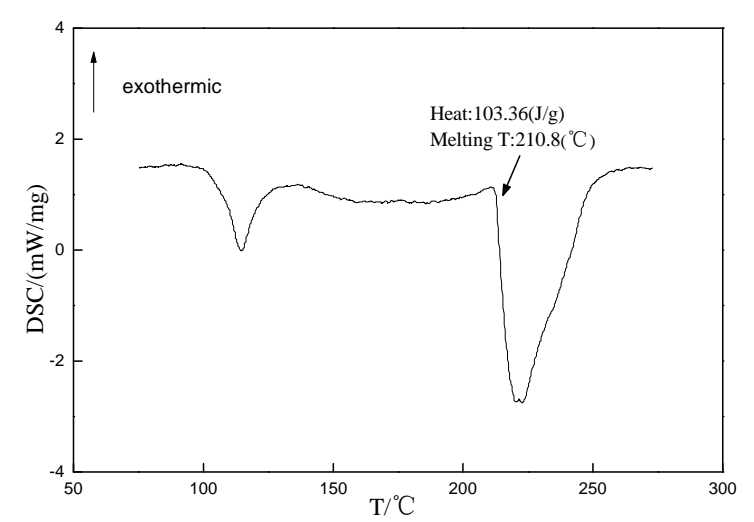

Figure 3. DSC curve of Solar Two+5\%additive-A

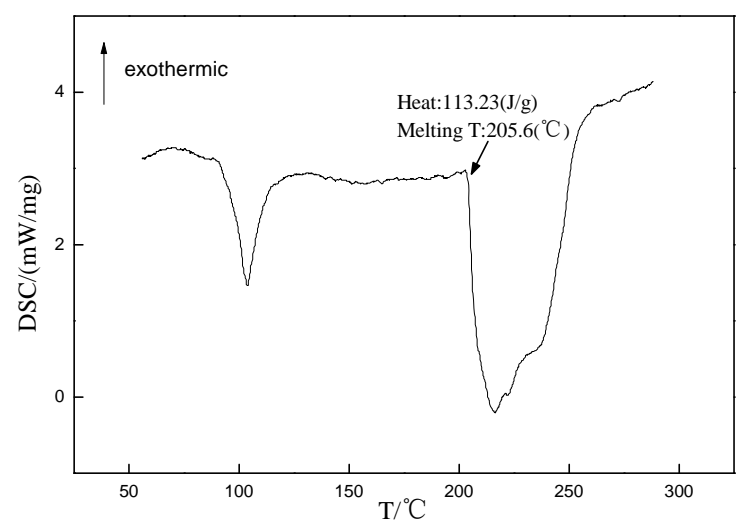

Figure 4. DSC curve of Solar Two+10\%additive-A

Figure 5 and 6 are the DSC curve of Solar Two $+5 \%$ additive- $\mathrm{B}, 10 \%$ additive-B.The figure shows that When adding $5 \%, 10 \%$ additive-B, the melting point of molten salts were lower, while the heat of fusion significantly reduced. When adding additive- $B$, it can decline the melting point, while also significantly reduce heat of fusion, the impact of the thermodynamic properties of molten salts. Thus, the additive-B has better modification effect in terms of lower the melting point of mixture molten salts.

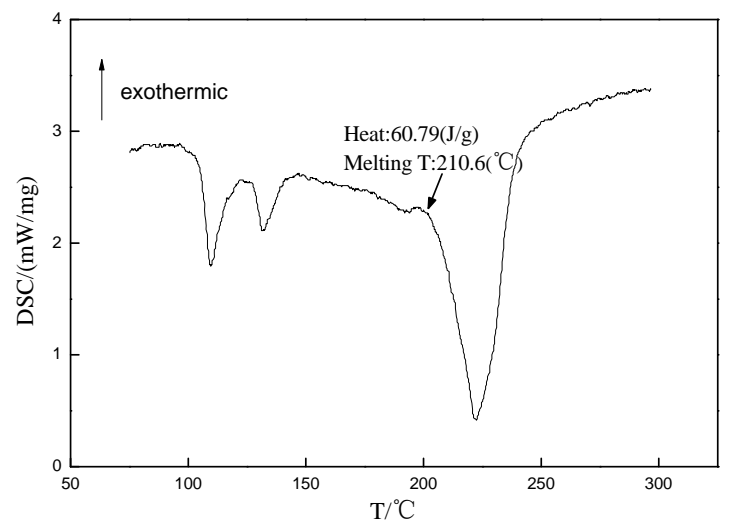

Figure 5. DSC curve of Solar Two+5\%additive-B

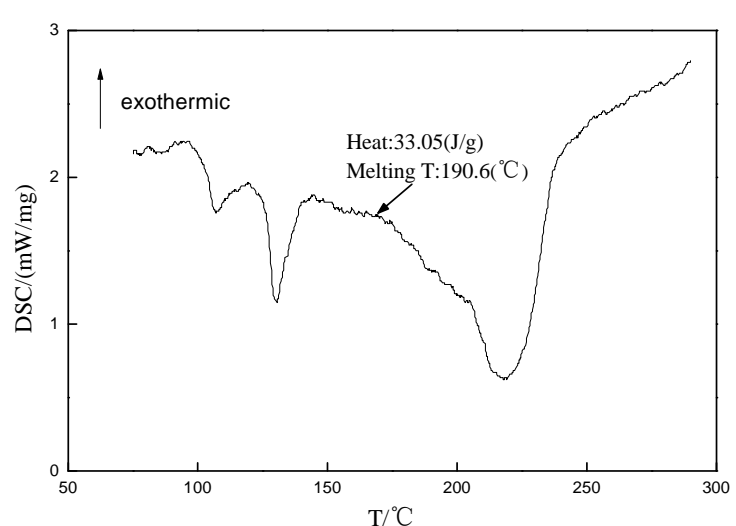

Figure 6. DSC curve of Solar Two+10\%additive-B

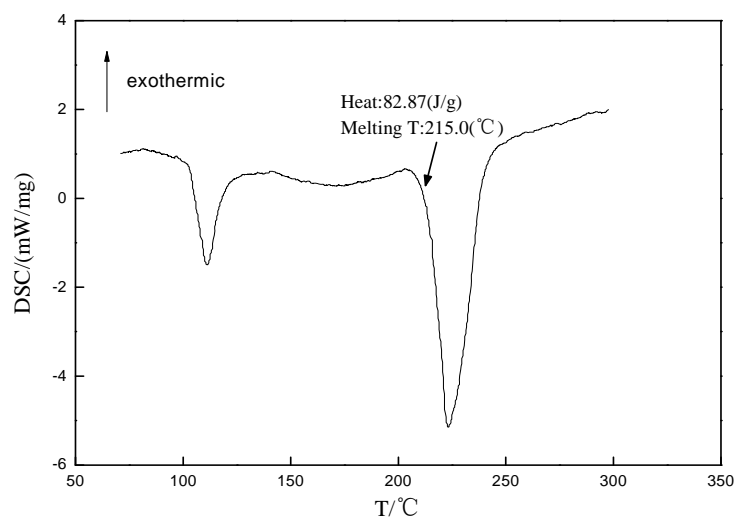

Figure 7. DSC curve of Solar Two+2\%additive-C

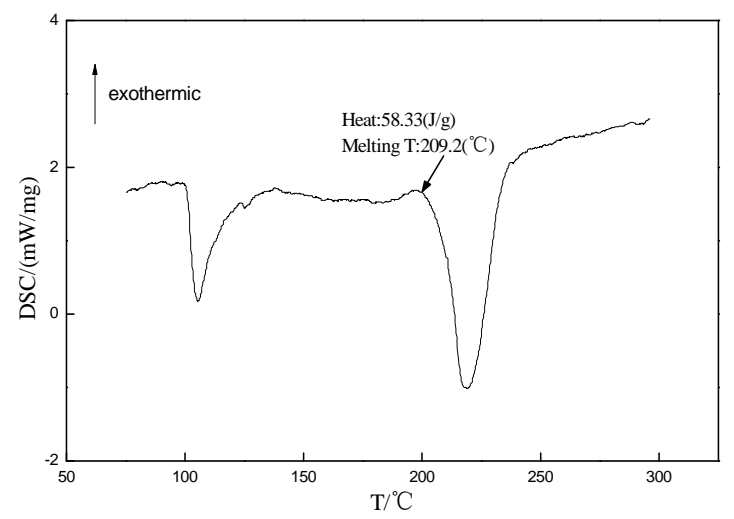

Figure 8. DSC curve of Solar Two+5\%additive-C

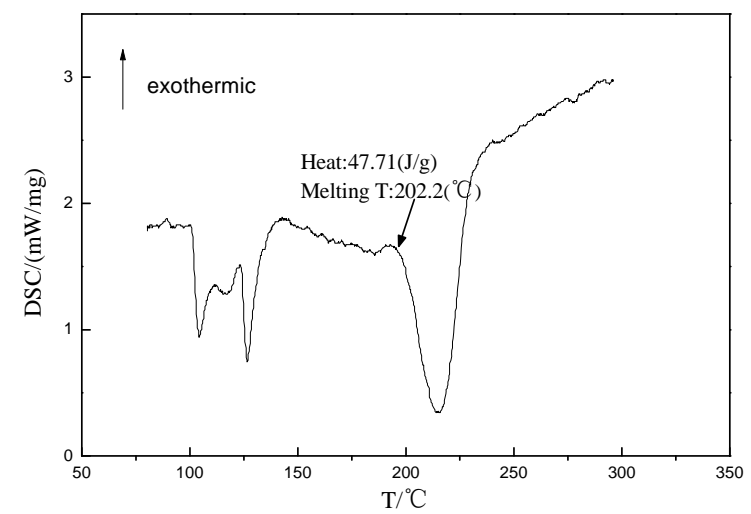

Figure 9. DSC curve of Solar Two+10\%additive-C 
Figure 7-9 shows the DSC curve of Solar Two $+2 \%$ additive-C, 5\%additive-C, 10\%additive-C. When adding additive-C, slightly lower the melting point of molten salts is slightly lower, heat of fusion is reduce. When added in an amount of 5\%,10\%, the heat of fusion of mixture molten salts is drama anxious reduced, it affects the thermodynamic properties of molten salt.

By analyzing the effect of adding three different additives, there are results that additive-A can optimize the melting point and heat of fusion of molten salts. Additive-B and Additive- $C$ have good effect only in melting point or melting heat unilaterally. Furthermore, the amount of $10 \%$ additive-A have optimum modification effect.

\subsection{Density of Solar Two+10\%additive-A molten salt}

Figure 10 shows the heat capacity curve of Solar Two+10\%additive-A molten salt. The heat capacity data for Solar Two+10\%additive-A was solid state in the temperature range of 66.85 to $216.85{ }^{\circ} \mathrm{C}$. The curve showed approximate linear increase with temperature increasing. The modified salt showed a relatively large specific heat capacity in the liquid state, which was suitable for the heat storage.

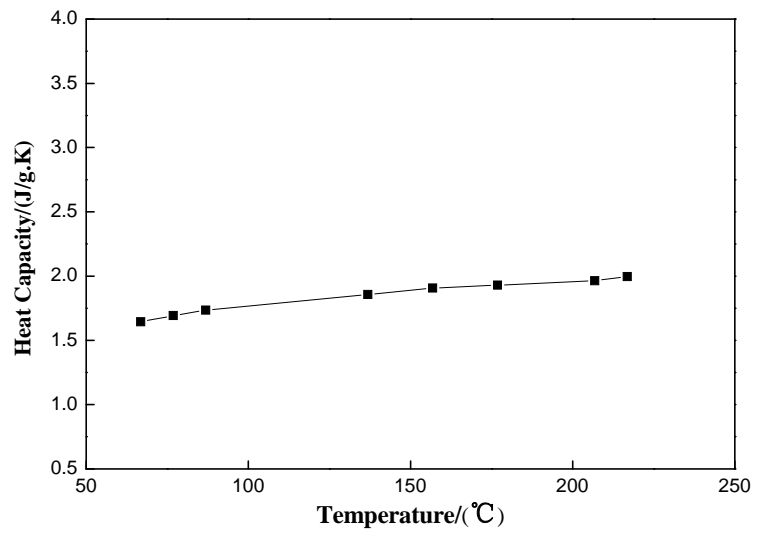

Figure 10. Heat capacity curve of Solar Two $+10 \%$ additive-A

The density of Solar Two+10\%additive-A molten salt as function of temperature is plotted in Figure 11. With the temperature increasing, the density of the molten salt would decrease. In the liquid state the density has linearly dependence with temperature[12-14], which can be expressed as $\mathrm{q}=\mathrm{A}$ - $\mathrm{Bt}$. The regression analysis is performed to determine the coefficients $\mathrm{A}$ and $\mathrm{B}$ of the modified solar two molten salt. The results obtained for A and $B$ are $2.1476 \mathrm{~g} / \mathrm{cm}^{3}$ and $0.7 \times 10^{-3} \mathrm{~g} / \mathrm{cm}^{3}{ }^{\circ} \mathrm{C}$, respectively.

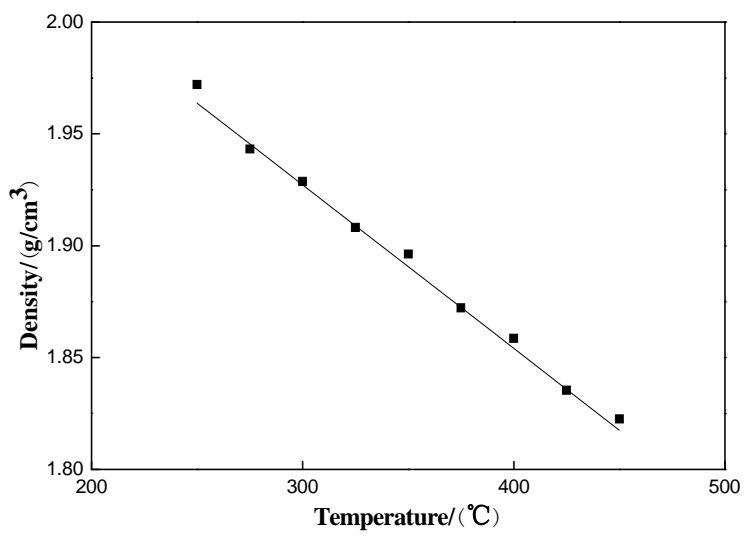

Figure 11. Density curve of Solar Two $+10 \%$ additive-A

\subsection{Viscosity and thermal conductivity of Solar Two+10\%additive-A}

Table 2 . The viscosity and thermal conductivity of the SolarTwo $+10 \%$ additive- $\mathrm{A}$ at $350^{\circ} \mathrm{C}$

\begin{tabular}{|c|c|c|}
\hline Molten & $\begin{array}{c}\text { Viscosity /10- } \\
\mathbf{3 P a} \cdot \mathbf{s}\end{array}$ & $\begin{array}{c}\text { Thermal } \\
\text { conductivity/W. } \mathbf{m} \\
\boldsymbol{- 1}^{-1} \cdot \mathbf{K}^{-\mathbf{1}}\end{array}$ \\
\hline $\begin{array}{c}\text { modified solar } \\
\text { two }\end{array}$ & 1.713 & 0.542 \\
\hline
\end{tabular}

The viscosity and thermal conductivity of Solar Two $+10 \%$ additive- $\mathrm{A}$ at $350^{\circ} \mathrm{C}$ is listed in Table 1 .The viscosity of Solar Two $+10 \%$ additive-A is about $1.8 \mathrm{Cp}$. The viscosity of the molten salt is less than $5 \mathrm{Cp}$, which showed that the molten salt had better epidemic.

The thermal conductivity of Solar Two $+10 \%$ additiveA is $0.542 / \mathrm{W} \cdot \mathrm{m}^{-1} \cdot \mathrm{K}^{-1}$. The thermal conductivity value is relatively large. It is suitable for heat heat/storage of solar thermal power system.

\section{Conclusions}

In this paper, by adding different additives in Solar Two molten salt in order to form multiple composite molten salt. By testing the thermal performance of mixture molten salt can be comparatively analysis of the modification effects of different additives. There are results that additive- $\mathrm{A}$ can optimize the melting point and heat of fusion of molten salts, Additive-B and Additive-C have good effect only in melting point or melting heat unilaterally. And he amount of $10 \%$ additive-A have optimum modification effect. Meanwhile, the Solar Two+10\%additive-A has lower melting point, higher latent heat, good density and higher specific heat capacity. Through the above experimental and analysis results can screen excellent additives which modify Solar Two molten Salt, thereby it improve the thermal performance of heat transfer and storage thermal material. 


\section{Acknowledgements}

This work was financially supported by heat transfer of energy-saving single tank thermocline molten salt thermal storage system and general administration of quality supervision, inspection and quarantine of the people's republic of china.

\section{References}

[1] Olivares Rene I. The thermal stability of molten nitrite/nitrates salt for solar thermal energy storage in different atmospheres[J]. Solar Energy, 2012,86(9): 2576-2583

[2] Johnson Winford B, Nagle Wesley M. Circulating HTS as a Pilot Plant Heat Transfer Medium[J]. Industrial \& Engineering Chemistry, 1947,39(8): 971-974

[3] Kearney D, Kelly Bruce, Herrmann Ulf, et al. Engineering aspects of a molten salt heat transfer fluid in a trough solar field[J]. Energy, 2004,29(5): 861-870

[4] Kamimoto Masayuki, Tanaka Tadayoshi, Tani Tatsuo ,et al. Investigation of nitrate salts for solar latent heat storage[J]. Solar Energy, 1980,24(6): 581587

[5] Raade J.W, Padowitz D. Development of molten salt heat transfer fluid with low melting point and high thermal stability[J]. Transactions of the ASME-NJournal of Solar Energy Engineering, 2011,133(3): 31013

[6] Xiangyang Shen, Jing Ding, Qiang Peng. Hightemperature molten salt used in solar thermal power generation[J]. Chemical Industry of Guangdong, 2007,34(11): 49-52

[7] Raade Justin W. Development of Molten Salt Heat Transfer Fluid With Low Melting Point and High Thermal Stability[J]. Journal of Solar Energy Engineering, 2011,133

[8] Mills David. Advances in solar thermal electricity technology[J]. Solar energy, 2004,76(1): 19-31

[9] Alexander Jr J, Hindin SG. Phase relations in heat transfer salt systems[J]. Industrial \& Engineering Chemistry, 1947,39(8): 1044-1049

[10]Kirst WE, Nagle WM, Castner JB. A new heat transfer medium for high temperatures[J]. Trans. Am. Inst. Chem. Eng, 1940,36(371

[11] Silverman M D, Engel J R. Survey of technology for technology for storage of thermal energy in heat transfer salt[R]. OakRidge National Lab, Report ORNL/TM-568, 1977, 2.

[12] Janz George J, Krebs Ursula, Siegenthaler HF, et al. Molten salts: Volume 3 nitrates, nitrites, and mixtures: Electrical conductance, density, viscosity, and surface tension data[J]. Journal of Physical and Chemical Reference Data, 1972,1(3): 581-746

[13]Nasch PM, Steinemann SG. Density and thermal expansion of molten manganese, iron, nickel, copper, aluminum and tin by means of the gamma-ray attenuation technique[J]. Physics and Chemistry of Liquids, 1995,29(1): 43-58

[14]Boardman NK, Dorman FH, Heymann E. Densities and molar volumes of molten salt mixtures[J]. The Journal of Physical Chemistry, 1949,53(3): 375-382 